\title{
DESCARGA DE ÁGUA SUBTERRÂNEA PARA AS LAGOAS COSTEIRAS DO LITORAL NORTE DO RIO GRANDE DO SUL, BRASIL
}

\author{
ROCHA, C.M. ${ }^{1,2 *}$; ANDRADE, C.F.F. ${ }^{1} \&$ NIENCHESKI, L.F.H. ${ }^{1}$ \\ 1. Laboratório de Hidroquímica/Instituto de Oceanografia/FURG \\ 2. Laboratório de Análise Águas/CECLIMAR/Instituto de Biociências/UFRGS \\ *Corresponding author: cacinele@gmail.com
}

\begin{abstract}
Rocha, C.M.; Andrade, C.F.F. \& Niencheski, L.F.H. (2015). Descarga de água subterrânea para as lagoas costeiras do Litoral Norte do Rio Grande do Sul, Brasil. Braz. J. Aquat. Sci. Technol. 19(2). elSSN 1983-9057. DOI: 10.14210/ bjast.v19n2. The advection of groundwater in coastal lakes of southern Rio Grande do Sul State is already a well known process. This article is the first one dealing with Submarine Groundwater Discharge (SGD) in the northern coast of state to confirm and quantify the SGD in four major lakes of this region - Itapeva, Quadros, Pinguela and Barros, applying seepage meter method. In each lake two sets of sampling stations were selected. A different behavior between margins was recorded. In the margins near the hills were identified advective process; and those close to the shore line, were dominated by infiltration process. The estimated volume of the SGD reaches the order of millions of cubic meters annually toward to these lakes, accounting for $13-27 \%$ of total contributions. Furthermore, utilizing water balance it was possible to evaluate the water balance between inputs and outputs as well as the residence time in each water bodies, which ranged from 19 to 847 days.
\end{abstract}

Keywords: Water balance, Seepage meter, Submarine groundwater discharge, Residence time.

\section{INTRODUÇÃO}

É comprovada a interligação hidráulica com os sistemas aquíferos sobrepostos (Rebouças, 1994), sendo que a descarga de água subterrânea, DAS (do inglês, Submarine Groundwater Discharge - SGD) é um fenômeno relativamente comum quando conectado às águas superficiais através de sedimentos permeáveis ou mesmo fissuras (Johannes, 1980).

Atualmente, esse processo foi reconhecido por Taniguchi et al. (2007), em relação ao impacto do fluxo de águas subterrâneas, sendo que Cable et al. (1996) e Lee (1977) observam que o mesmo cresce exponencialmente com a proximidade da costa. Assim, podemos ter como base que estes aportes subterrâneos respondem por significativa parcela do abastecimento lacustre e costeiro.

Estudos anteriores realizados na região sul do estado do Rio Grande do Sul (RS) (Andrade, 2010), apontam para divergências nos valores de balanço hidrológico de algumas lagoas que, posteriormente, foram investigados, resultando em taxas positivas de descarga subterrânea. A hipótese é de que essa situação pudesse se repetir também na região norte da planície costeira, possibilitando a identificação de fontes de água subterrânea nos sistemas de lagoas do Litoral Norte do Estado.

A planície costeira do Rio Grande do Sul foi formada, durante o Quaternário, através de sistemas de leques aluviais sendo seguidos por contínuos eventos de regressão e transgressão marinha, os quais deram origem aos sistemas Laguna/Barreira I, II, III e IV (Tomazelli, 1990; Villwock \& Tomazelli, 1995; Tomazelli \& Villwock, 2005; Tomazelli et al., 2011; Rosa, 2012; Dillenburg \& Barboza, 2014). No Litoral Norte, esses processos deram início à formação das dezenas de lagoas cordiformes (Tomazelli \& Villwock, 2005), como Itapeva, Quadros e Barros (Figura 1), que representam $38,5 \%$ da superfície local (Schwarzbold \& Schafer, 1984), sendo a maioria dessas lagoas de pouca profundidade, mais rasas na margem oeste pelo avanço do sistema eólico (Tomazelli, 1993), interligadas entre si (Delaney, 1965) e que atuam como filtros daquilo que alcança o oceano, podendo apresentar taxa de sedimentação de alguns milímetros por ano (Ivanoff, 2013), e que estão situadas entre a encosta e a linha de praia.

O embasamento, sobre o qual se estabelece esta área, é formado por rochas da Bacia Sedimentar do Paraná, estabelecida sobre a placa continental no interior cratônico do Gondwana, e que sofreu vários processos deposicionais, em períodos de soerguimento e erosão que se revezaram (Rosa Filho et al., 2003; Silva et al., 2003; Milani et al., 2007).

Uma das metodologias para detecção da ocorrência da DAS é o uso do seepage meter, descrito por Lee (1977). Este método de avaliação da advecção foi empregado em diversos trabalhos (Shaw \& Prepas, 1989; Cable et al., 1997a; Corbett et al., 1999), por sua facilidade de aplicação, resposta rápida e baixos cus- 
tos envolvidos, sendo os resultados preliminarmente importantes para qualificar e quantificar volumes que advectam em pontos específicos.

\section{MATERIAL E MÉTODOS}

Dentre as mais de 60 lagoas costeiras do Litoral Norte do Rio Grande do Sul, foram escolhidas quatro delas por sua importante relevância regional, seja pelos seus usos no abastecimento e na agricultura, ou mesmo por seu volume e área estabelecidos.

Foram definidos quatro pares de pontos de amostragem, sendo um par a cada lagoa, de forma que cada um dos pontos estivesse o mais distante possível um do outro. Localizados, preferencialmente, nas porções próximas ao embasamento e próximo da linha da praia para avaliação de possíveis diferenças no comportamento no fluxo, conforme Figura 1.

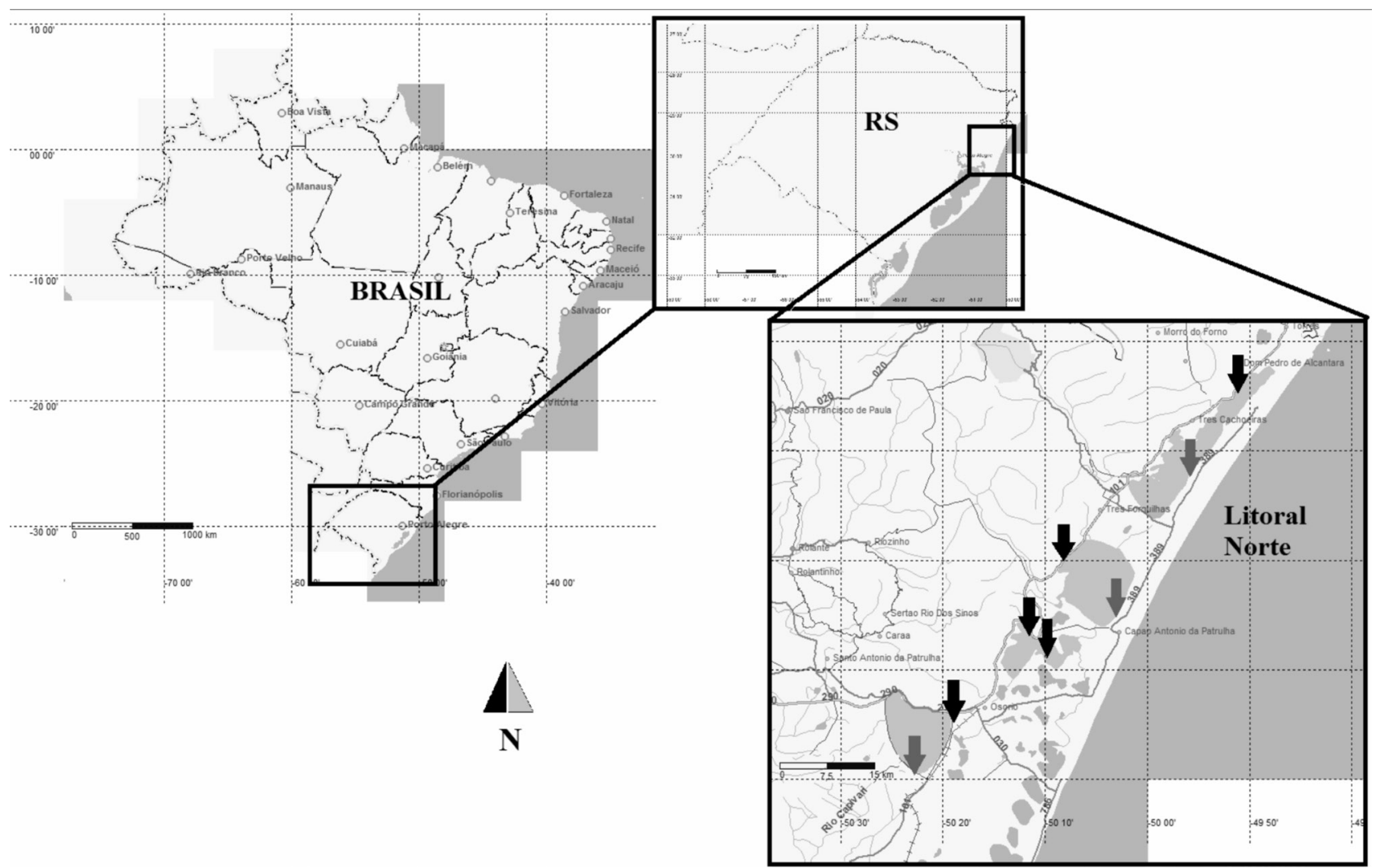

Figura 1 - Mapa da área de estudo no Litoral Norte do RS, Brasil, e em destaque pontos de instalação do seepage meter.

Cada ponto de aplicação do método esteve composto de três sistemas posicionados equidistantes entre si. A avaliação em ambas as margens de cada lagoa foi executada num mesmo período para que todos os seepage meter estivessem expostos às mesmas condições de tempo e gradiente hidráulico.

Considerando que os processos de infiltração e advecção são: "movimento da água dentro do solo" (Guerra \& Cunha, 1994), sem um sentido definido e com transferência de massa, optamos por estabelecer a nomenclatura "infiltração" para o movimento no sentido compartimento superficial $\rightarrow$ manancial subterrâneo, enquanto para o termo "advecção" consideramos o movimento do manancial subterrâneo $\rightarrow$ compartimento superficial. Esta definição deve-se a necessidade corriqueira de informar o processo indicando o sentido de movimento, importante característica abordada neste trabalho.

\section{Seepage meter}

O sistema foi adaptado e confeccionado de forma simples, usando um recipiente emborcado e penetrado no sedimento por cerca de $20 \mathrm{~cm}$, tendo em sua extremidade superior uma válvula, onde foi preso um saco plástico com volume conhecido de água (Figura 2). $\mathrm{O}$ mesmo permaneceu submerso por um período aproximado de 24 horas e, neste intervalo, o sistema teve chance de perder volume por infiltração ou ganhar pelo processo de advecção de água subterrânea (Lee, 1977).

Considerando a área de exposição e o volume de água remanescente no saco plástico, em relação 


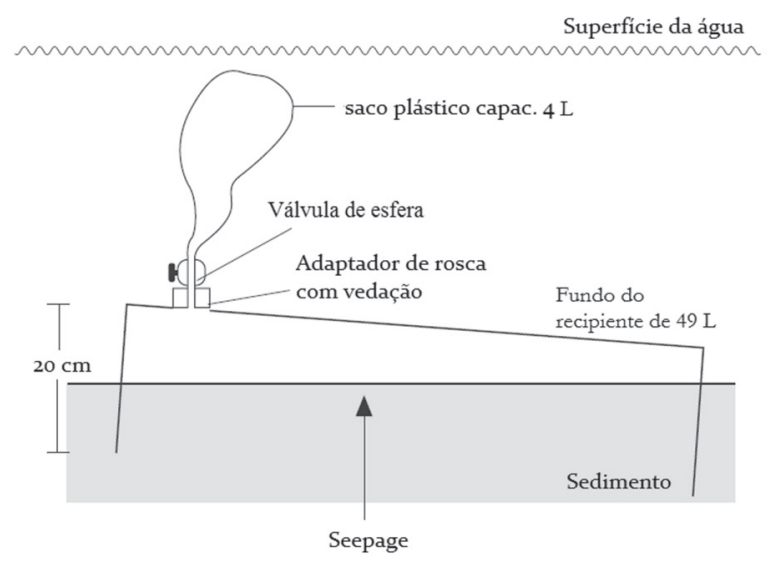

Figura 2 - Esquema seepage meter, empregado na avaliação dos movimentos de água entre superfície e subsuperfície, através de um recipiente fixado ao sedimento e exposto por determinado período de tempo.

ao tempo de exposição, foi possível obter uma estimativa do volume de água que escoou através do sedimento, movimentando-se entre superfície e subterrâneo.

O cálculo para obter a taxa do processo advectivo é dado pela Equação I:

$$
\mathrm{Tx}=\frac{(\mathrm{m} \times 100)}{1000000 \times \Delta \mathrm{txA}_{\mathrm{r}}}
$$

onde:

Tx - taxa de advecção ou infiltração, dada em $\mathrm{cm} \mathrm{d}^{-1}$

m - média dos volumes acumulados (+) ou perdidos (-) durante o experimento, acompanhados do sinal, expresso em litros;

$\Delta t$ - tempo decorrido entre a instalação e a retirada dos sistemas, ou seja, tempo de exposição, dado em dias, d; $\mathrm{m}^{2}$.

$A_{r}$ - área dos recipientes, expresso em metros,

Sendo que $\mathrm{m}$ pode ser calculado através da Equação II:

$$
\mathrm{m}=\frac{\left[\left(\mathrm{V}_{\mathrm{i}}-\mathrm{V}_{\mathrm{f}}\right)_{\mathrm{s} 1}+\left(\mathrm{V}_{\mathrm{i}}-\mathrm{V}_{\mathrm{f}}\right)_{\mathrm{s} 2}+\left(\mathrm{V}_{\mathrm{i}}-\mathrm{V}_{\mathrm{f}}\right)_{\mathrm{s} 3}\right]}{3}
$$

onde: litros;

$V_{i}$ - volume inicial do saco plástico, dado em litros;

$V_{f}$ - volume final do saco plástico, dado em

$s 1$ - referente ao saco do recipiente $n^{0} 1$;

s2 - referente ao saco do recipiente $n^{\circ} 2$;

s3 - referente ao saco do recipiente $n^{0} 3$.
Para a correta instalação do equipamento, fazse necessário que o local escolhido tenha característica arenosa, pouco lamosa e isenta de pedras. Desta forma, os pontos selecionados, conforme Tabela 1, foram:

Tabela 1 - Localização $\left(\mathrm{gg}^{\circ} \mathrm{m} . \mathrm{mmm}\right)$ dos pontos amostrados por lagoa.

\begin{tabular}{|c|c|c|c|c|}
\hline \multirow{2}{*}{ Lagoa } & \multicolumn{2}{|c|}{ Porção Embasamento } & \multicolumn{2}{c|}{ Porção Bacia do Paraná } \\
\cline { 2 - 5 } & Latitude & Longitude & Latitude & Longitude \\
\hline Itapeva & $29^{\circ} 24.378^{\prime} S$ & $49^{\circ} 50.994^{\prime} \mathrm{O}$ & $29^{\circ} 32.192^{\prime} \mathrm{S}$ & $49^{\circ} 55.836^{\prime} \mathrm{O}$ \\
\hline Quadros & $29^{\circ} 40.650^{\circ} \mathrm{S}$ & $50^{\circ} 08.652^{\prime} \mathrm{O}$ & $29^{\circ} 45.218^{\prime} \mathrm{S}$ & $50^{\circ} 02.937^{\prime} \mathrm{O}$ \\
\hline Pinguela & $29^{\circ} 46.838^{\prime} \mathrm{S}$ & $50^{\circ} 11.467^{\prime} \mathrm{O}$ & $29^{\circ} 49.158^{\prime} \mathrm{S}$ & $50^{\circ} 10.077^{\prime} \mathrm{O}$ \\
\hline Barros & $29^{\circ} 54.076^{\prime} \mathrm{S}$ & $50^{\circ} 19.276^{\prime} \mathrm{O}$ & $29^{\circ} 59.386^{\prime} \mathrm{S}$ & $50^{\circ} 22.640^{\prime} \mathrm{O}$ \\
\hline
\end{tabular}

\section{RESULTADOS}

\section{Seepage meter}

A coleta de dados ocorreu em período com precipitação média mensal de $209 \mathrm{~mm}$ (máxima: $432 \mathrm{~mm}$; mínima: 73,4 mm) (INMET, 2013), entre os meses de agosto de 2013 e fevereiro de 2014, e os resultados variaram desde $-0,16 \mathrm{~cm} \mathrm{~d}^{-1}$, ou seja, perda por infiltração de água, e $+0,70 \mathrm{~cm} \mathrm{~d}^{-1}$, indicando a DAS (Figura 3). Os mesmos estão esquematicamente apresentados na Figura 1, onde os fluxos de infiltração estão representados em cinza e, os de advecção em preto.

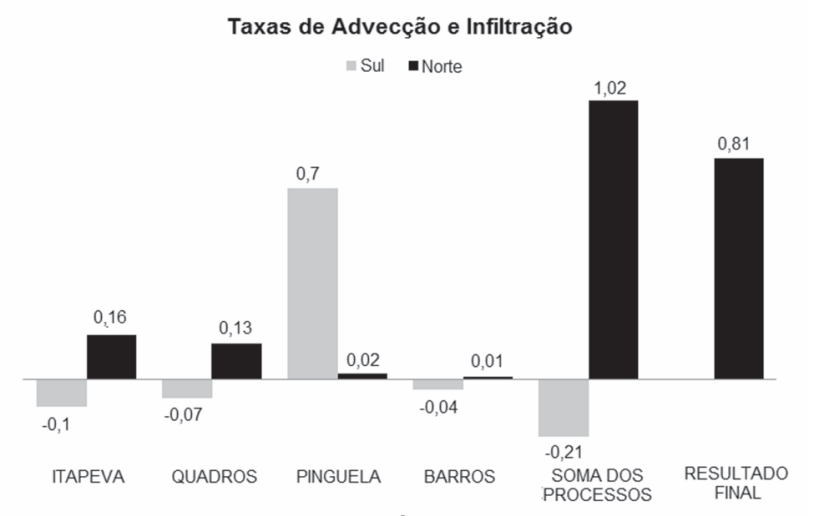

Figura 3 - Representação gráfica dos processos advectivos, positivos, e infiltrante, negativos, nas lagoas amostradas em suas porções opostas e, somatórios dos processos de todo sistema.

Assim, de acordo com as áreas totais das lagoas (Tabela 2) e as taxas encontradas para ambos os pontos avaliados, foi possível calcular os volumes anuais advectados ou infiltrados, conforme a Equação III:

$$
\mathrm{VT}_{\mathrm{a}}=\frac{\mathrm{T}_{\mathrm{x}} \times \mathrm{A}_{\mathrm{l}} \times 365}{100}
$$

onde:

$\mathrm{VT}_{\mathrm{a}}$ - volume total por ano, dado por $\mathrm{m}^{3} \mathrm{ano}^{-1}$; 

$\mathrm{cm} \mathrm{d}^{-1}$;

Tx - taxa de advecção ou infiltração, dada em

$A_{1}$ - área do corpo lagunar, expresso em $\mathrm{m}^{2}$, sendo a mesma para o cálculo de ambos os pontos, em processo advectivo e/ou infiltrante.

Para todas as margens das lagoas próximas a elevação da Serra Geral foram registrados valores positivos, enquanto para as margens mais próximas da faixa de praia, foram registrados três resultados negativos e um positivo, conforme dados da Tabela 2.

Tabela 2 - Áreas lagunares, em km², e volumes anuais, em m3 106, dos processos advectivo (DAS) e infiltrante para as respectivas lagoas amostradas.

\begin{tabular}{|c|c|c|c|c|}
\hline Dados/Lagoas & Itapeva & Quadros & Pinguela & Barros \\
\hline Área & 194 & 373 & 107 & 124 \\
\hline Vol. advectado & $+71,5$ & +176 & $+275,6$ & $+48,6$ \\
\hline Vol. Infiltrado & $-113,4$ & $-101,5$ & $+8,2$ & $-16,5$ \\
\hline
\end{tabular}

\section{DAS e outros aportes}

A significância do aporte subterrâneo pode ser avaliada comparando-o com as demais entradas de água em cada um dos sistemas. A participação dos rios, canais, da precipitação e da DAS pode ser observado na Figura 4, com base nos dados da Tabela 1 , provenientes do Plano de Bacia da respectiva região (Profill, 2005).

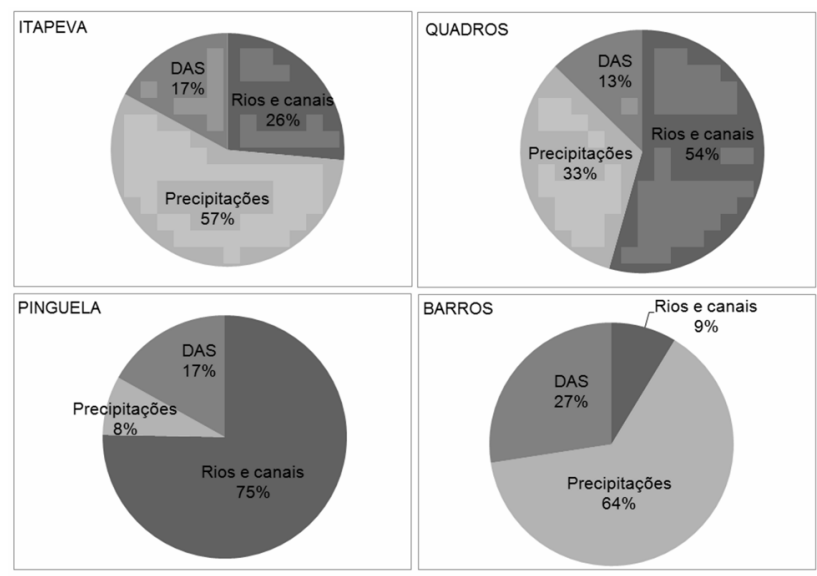

Figura 4 - Representação das parcelas de aporte de águas, classificados como tributários, águas provenientes de precipitações e de descarga de água subterrânea, para o período de um ano.

\section{Balanço hidrológico}

De posse dos valores de advecção e infiltração foi possível complementar os dados para avaliação do balanço hidrológico das lagoas estudadas, inclusive avaliando se os mesmos expressam equilíbrio ou não. Vale salientar que mesmo a aplicação da técnica seepage meter não tendo sido executada em paralelo, ou seja, os oito pontos simultaneamente, tivemos precipitação e níveis das lagoas com poucas variações, expondo os sistemas a características bastante semelhantes durante o período avaliado.
Como um balanço é o resultado da quantidade de água que entra e sai num dado volume de solo (Gomes, 2005), neste caso o espaço da lagoa, para um determinado intervalo de tempo, outros dados além da advecção/infiltração avaliados neste trabalho, foram necessários. Para tanto, consideramos seus respectivos volumes, também a precipitação e evaporação para o período (INMET, 2013), vazão dos rios, canais tributários e dos efluentes, bem como os volumes de retirada de água para os tantos usos, como abastecimento, irrigação, mineração, entre outros provindos dos relatórios de Plano de Bacia (Profill, 2005). Neste estudo, para uma melhor representatividade, optamos por um balanço em escala anual. Matematicamente, a estruturação do balanço hidrológico é dada por entradas e saídas, como na Equação IV:

$\sum$ anual=TRIB + PREC + DAS-EVAP-ESCOA-USO-INF $\quad($ Eq. IV)

onde:

$\sum$ anual $=$ somatório de circulação de águas para o período de um ano, em $\mathrm{m}^{3}$;

TRIB = volume de água recebida por meio de tributários no ano, em $\mathrm{m}^{3}$;

PREC = volume de água que precipita sobre a lagoa considerando sua área, expresso $\mathrm{em} \mathrm{m}^{3} \mathrm{ano}^{-1}$;

DAS = volume de água que advecta do subterrâneo para lagoa, dada $\mathrm{em} \mathrm{m}^{3} \mathrm{ano}^{-1}$, calculado através da Equação III, indicado por um VTa positivo;

EVAP = volume de água que evapora da lagoa considerada sua área, expresso $\mathrm{em} \mathrm{m}^{3} \mathrm{ano}^{-1}$;

$\mathrm{ESCOA}=$ volume de água que escoa para jusante da lagoa por meio de rios ou canais no período de um ano, em $\mathrm{m}^{3}$;

USO = volume de água que é retirada da lagoa anualmente para usos diversos, expresso em $\mathrm{m}^{3} \mathrm{ano}^{-1}$;

$\mathrm{INF}=$ volume de água que infiltra retornando da lagoa para o manancial subterrâneo, dado em $\mathrm{m}^{3}$ $a^{-1}{ }^{-1}$ calculado através da Equação III, indicado por um VTa negativo.

A variável escoamento superficial foi suprimida dos cálculos uma vez que não estavam disponíveis para estabelecimento do mesmo.

As componentes e o resultado para o cálculo do balanço hidrológico das lagoas estão indicados na Tabela 3.

Outro dado relevante para a gestão e compreensão dos sistemas lacustres de uma bacia hidrográfica é o tempo de residência (TR) de sua massa d'água (Equação $V$ ), isso porque reflete o trânsito de água no sistema, assim como os nutrientes carreados e o impacto sobre o ambiente local (Santos et al., 2008a). Conforme Sassaki (2005), o TR pode ser 
definido como "o tempo decorrido desde a sua entrada até sua saída no equipamento" ou sistema.

Tabela 3 - Resumo do balanço hidrológico, em escala anual, para as lagoas da Itapeva, dos Quadros, da Pinguela e dos Barros, indicando cada um dos fatores envolvidos com valores em $\mathrm{m}^{3} 10^{6}$, e o tempo de residência (TR), expresso em dias.

\begin{tabular}{|c|c|c|c|c|c|c|c|c|c|}
\hline Lagoa & TRIB & PREC & DAS & EVAP & ESCOA & USO & INF & Eanual & TR \\
\hline \multirow{2}{*}{ Itapeva } & $\begin{array}{c}\text { Três } \\
\text { Forquilhas } \\
88,5\end{array}$ & \multirow[t]{2}{*}{236} & \multirow[t]{2}{*}{71,5} & \multirow{2}{*}{158} & \multirow{2}{*}{$\begin{array}{c}\text { Cornélios } \\
587\end{array}$} & \multirow[t]{2}{*}{2,9} & \multirow[t]{2}{*}{-113} & \multirow[t]{2}{*}{$-443,2$} & \multirow[t]{2}{*}{105} \\
\hline & $\begin{array}{c}\text { Cardoso } \\
22,1\end{array}$ & & & & & & & & \\
\hline \multirow[b]{2}{*}{ Quadros } & $\begin{array}{c}\text { Maquiné } \\
165\end{array}$ & \multirow[b]{2}{*}{455} & \multirow[b]{2}{*}{176} & \multirow[b]{2}{*}{304} & \multirow{2}{*}{$\begin{array}{c}\text { João } \\
\text { Pedro } \\
1264\end{array}$} & \multirow[b]{2}{*}{11,9} & \multirow[b]{2}{*}{$-101,5$} & \multirow[b]{2}{*}{$-413,4$} & \multirow[b]{2}{*}{66} \\
\hline & $\begin{array}{c}\text { Tornélios } \\
587 \\
\end{array}$ & & & & & & & & \\
\hline \multirow[b]{2}{*}{ Pinguela } & $\begin{array}{l}\text { João } \\
\text { Pedro } \\
1264\end{array}$ & \multirow[b]{2}{*}{131} & \multirow[b]{2}{*}{275,6} & \multirow[b]{2}{*}{90} & \multirow{2}{*}{$\begin{array}{l}\text { Dos } \\
\text { Postes } \\
1331\end{array}$} & \multirow[b]{2}{*}{0,09} & \multirow[b]{2}{*}{$+8,2$} & \multirow[b]{2}{*}{$+257,4$} & \multirow[b]{2}{*}{19} \\
\hline & $\begin{array}{l}\text { Ligação } \\
\text { Peixoto }\end{array}$ & & & & & & & & \\
\hline Barros & $\begin{array}{c}\text { Córrego } \\
15,4\end{array}$ & 113 & 48,6 & 78,4 & - & 352,3 & $-16,5$ & $-269,8$ & 847 \\
\hline
\end{tabular}

De acordo com os cálculos descritos anteriormente, os balanços hidrológicos não demonstraram estar equilibrados porque os valores aqui verificados para a DAS e infiltração possivelmente sejam uma representação ainda reduzida da área. Ainda assim, para o cálculo da TR é possível usar dados de saídas ou aportes, sendo que optamos por este último como é demonstrado a seguir:

$$
\mathrm{TR}=\frac{\mathrm{Vt} \times 365}{\mathrm{TRIB}+\mathrm{PREC}+\mathrm{DAS}}
$$

onde:

$\mathrm{TR}=$ tempo de residência das águas das lagoas, dada em dias;

$\mathrm{Vt}=$ volume total da lagoa, definida por sua área e profundidade, expressa $\mathrm{em}^{3}$;

TRIB = volume de água recebida por meio de tributários no ano, em $\mathrm{m}^{3}$;

PREC = volume de água que precipita sobre a lagoa considerando sua área, expresso em $\mathrm{m}^{3}$ ano-1; $^{-1}$

DAS = volume de água que advecta do subterrâneo para lagoa, dada $\mathrm{em} \mathrm{m}^{3} \mathrm{ano}^{-1}$, calculado através da Equação III, indicado por um Vt positivo.

Desta forma, o TR das águas nas lagoas da Itapeva, dos Quadros, da Pinguela e dos Barros seria de poucas semanas até alguns meses, conforme Tabela 3.

\section{DISCUSSÃO}

Foi possível verificar um comportamento diferenciado entre as margens dentro de uma mesma lagoa quando aplicada à técnica seepage meter. Aqueles pontos próximos da encosta da Serra Geral demonstram um potencial advectivo maior, enquanto os pontos das margens mais próximas da linha de praia caracterizam-se como infiltrantes. Andrade (2010) também observou diferença entre as margens leste e oeste para a Lagoa Mangueira. Possivelmente, este comportamento em nossa grade amostral possa ser explicado na encosta da serra, pela interação com a formação geológica, através de fraturas ou porções permeáveis sobre aquíferos confinados em terra (Corbett et al., 1999). Enquanto isso, a porção leste da área de estudo sofre efeito da barreira holocênica, parte progradante parte retrogradante (Barboza et al., 2011; Dillenburg \& Barboza, 2014) que a comunica com o oceano, configurando uma membrana arenosa permeável, como o caso da Lagoa Mangueira (Andrade, 2010). Esta barreira apresenta diferentes estruturas deposicionais ao longo de sua formação, ora transgressivas, ora regressivas ou agradantes (Hesp et al., 2005; Barboza et al., 2011) que influenciam diretamente na descarga de água subterrânea em direção ao mar, criando nesta área uma zona de mistura (Attisano, 2008).

Resultados observados em outros trabalhos aplicados nos Estados Unidos da América com seepage meter "tipo Lee", registraram taxas na faixa de 5 a $45 \mathrm{~cm} \mathrm{~d}^{-1} \mathrm{em}$ baías de Massachusetts (Mulligan \& Charette, 2006), 0 a $20 \mathrm{~cm} \mathrm{~d}^{-1}$ na Flórida (Cable et al., 1997b), 1 a $490 \mathrm{~cm} \mathrm{~d}^{-1}$ nas llhas Maurício (Burnett et al., 2006) e 0 a $12 \mathrm{~cm} \mathrm{~d}^{-1}$ para zonas costeiras de Minnesota e Wisconsin (Lee, 1977), enquanto para lagoas a faixa registrada esteve entre 0 e $22 \mathrm{~cm} \mathrm{~d}^{-1} \mathrm{em}$ Carolina do Norte e Nova Escócia (Lee, 1977). Desta forma, os resultados verificados no presente trabalho podem ser considerados baixos, mesmo havendo poucas referências para áreas lacustres. Entretanto, já são responsáveis por contribuir com volumes na casa de milhões de metros cúbicos ao ano para as referidas lagoas.

Vale destacar que, embora o comportamento entre margens possa ter se mostrado diferenciado, assumimos que os processos de advecção e infiltração são homogêneos para toda a lagoa, ou seja, ocorrem ambos em toda a extensão do corpo lagunar. Não seria prudente fracionar áreas com, hipoteticamente, indicação de um ou outro processo, sem uma extensa avaliação de áreas mais restritas, sob pena de incorrer em erros.

Conforme Tabela 2, os resultados dos pares de pontos por lagoa evidenciam a transferência de água no sentido manancial subterrâneo/lagoa - lagoa/ manancial subterrâneo próximo ao mar e a magnitude destes processos, com possível descarga na região de praia, já registrado por Paiva (2011) através de poços piezométricos amostrados na região de Torres, litoral norte do Rio Grande do Sul.

Ainda, Paiva (2011) empregando outra técnica de avaliação com o traçador radônio, encontrou 
valores aproximados de $1,5 \mathrm{~cm} \mathrm{~d}^{-1}$ para Lagoa da Itapeva; 19,9 $\mathrm{cm} \mathrm{d}^{-1}$ para Lagoa dos Quadros; e 0,9 cm $\mathrm{d}^{-1}$ para Lagoa da Pinguela, resultados mais elevados que os encontrados com o uso do seepage meter. Segundo Burnett et al. (2003), essas descargas de águas provindas do subterrâneo oscilam muito, sendo variáveis com o tempo, desiguais e difusas em vista de múltiplas forças. Desta forma, quanto mais variadas as técnicas de verificação deste aporte, maiores as chances de cobrir significativo percentual destas manifestações.

Além das diferenças observáveis no sentido de escoamentos das águas, seja manancial subterrâneo/ lagoa ou lagoa/manancial subterrâneo, nota-se ainda que o processo positivo de advecção é duas a três vezes maior que o processo inverso, exceto para a Lagoa da Itapeva. De acordo com Pivel \& Calliari (1998), a característica marcante na região do litoral de Torres, onde localiza-se parte da Lagoa da Itapeva, é a ocorrência do contato das rochas da Bacia do Paraná, diretamente com o oceano (Rosa, 2012), assim como do afloramento do Sistema Aquífero Guarani - SAG (Rosa Filho et al., 2003) que poderia interferir positivamente na descarga de água subterrânea diretamente no mar, favorecendo uma maior taxa de transferência lagoa/manancial subterrâneo/oceano, justificando esta diferença quando comparada com as demais.

As oscilações entre os valores advectados/infiltrados entre as lagoas, não apresentam clara relação com suas respectivas áreas. Entretanto, as lagoas que apresentaram volumes mais elevados de DAS, como a Itapeva, Quadros e Pinguela, podem ter explicação na proximidade com o sistema de falhas Terra de Areia - Posadas, do Sistema Aquífero Guarani (Machado \& Freitas (2005), o qual favoreceria a advecção e do qual a Lagoa dos Barros está mais distante. Machado \& Freitas (2005) estudaram exaustivamente o SAG, tendo produzido um mapa hidrogeológico para o Rio Grande do Sul, caracterizando as diferentes regiões deste manancial. Esta região litorânea foi dividida em dois sistemas, Aquífero Quaternário Costeiro I, sob domínio de depósitos sedimentares de camadas inconsolidadas que se estende do Chuí a Torres, e esta área também foi classificada geologicamente como sistema Laguna/Barreira IV, de origem holocênica (Tomazelli, 1990). O segundo sistema hidrológico é o Aquífero Quaternário Costeiro II, também composto por sedimentos inconsolidados que se estende de Santa Vitória do Palmar a Torres, restringindo-se a região lagunar interna e os contrafortes da Serra Geral (Machado \& Freitas, 2005). Este último coincide com a Barreira III do sistema geológico Laguna/Barreira, de origem pleistocênica (Tomazelli, 1990). Esta conformação pode explicar o comportamento a respeito dos processos e forçantes envolvidas. Nas áreas das lagoas observa-se que o SAG, ou aquífero Botucatu, é parte livre e parte confinado (Rebouças, 1994), podendo ser esta a justificativa para diferenças entre os pontos avaliados. Já o Aquífero Serra Geral III também está representado nesta região de estudo, restringindo-se às porções mais elevadas dos derrames da Serra Geral e que pode, da mesma forma, influenciar estes sistemas pela proximidade, especialmente nas regiões dos rios, apresentando fluxos por meio de fraturas/fissuras das superfícies de descontinuidade horizontal, aqui estabelecidas pela região de encosta (Machado \& Freitas, 2005).

Conforme os resultados da Figura 4, sobre a participação da DAS nas entradas de águas nas lagoas, o expressivo aporte via tributários fica evidente, assim como para outros corpos; que as precipitações são mais representativas em vista de sua área ampla, favorecendo o acúmulo de um significante volume de águas. Para a Lagoa dos Barros, o papel dos tributários é bastante reduzido quando comparado às demais lagoas, considerando a inexistência de expressivos rios afluentes desta. Não menos importantes é a DAS, que demonstrou representar parte expressiva nestes sistemas. Ainda que o percentual seja menor que as demais fontes de água, as águas de origem subterrânea têm uma característica marcante que engrandece sua participação, que é a sua composição química. A quantidade de nutrientes é muitas ordens de magnitude maior se comparadas às águas de fontes superficiais ou provenientes de chuvas (Lee, 1977; Valiela et al., 1978; Zimmermann et al., 1985; Valiela \& D'Elia, 1990; Oberdorfer et al., 1999). Andrade (2010), inclusive, evidenciou a significativa contribuição de nutrientes associada à DAS na Lagoa Mangueira, no sul do Brasil, sendo que estes nutrientes são reciclados inúmeras vezes sustentando a produção primária de forma contínua, assim como também são importantes quando inseridos na plataforma continental (Corbett et al., 1999; Niencheski et al., 2007; Attisano et al., 2008; Santos et al., 2008b; Andrade et al., 2011).

Este processo é importante para a produtividade biológica e até mesmo em processos de remineralização geológica (Simmons, 1992). Em 2006, Mulligan \& Charette descreveram este processo como fonte principal de nutrientes em diversos estuários e baías, de considerável efeito ecológico, enquanto que Corbett et al. (1999) estimaram que essa contribuição chega a ser aproximadamente igual à contribuição via escoamento superficial em alguns locais. Ainda, outros autores afirmaram que a descarga decresce fortemente com a distância da costa, sendo que de 40 a $98 \%$ da descarga ocorreria até $100 \mathrm{~m}$ da costa (Johannes, 1980), entretanto, estudos mais recentes complementam estas informações, afirmando que 
essas advecções podem ocorrer bem longe da costa, inclusive na porção continental (Corbett et al., 1999), como em margens lagunares, como as mencionadas neste estudo que chegam a estar mais de $22 \mathrm{Km}$ adentro continente, distantes da faixa de praia.

Para todos os balanços hidrológicos avaliados, o somatório de volume foi, para o período de um ano, diferentes de zero, número que seria o esperado já que um balanço segue o princípio da conservação de massas (Gomes, 2005). Estes resultados podem sofrer uma variabilidade na contabilização dos dados empregados, bem como podem denotar que a normalização dos dados de forma anual, período de tempo empregado, pode não ser o mais correto. Para a natureza, a equalização de balanço de uma lagoa pode se dar naturalmente em períodos diferentes de 365 dias. Ainda, todas as entradas e saídas de um sistema são suscetíveis às mudanças e fenômenos climáticos, como El Niño e La Niña. Esses também influenciam os usos de água, como por exemplo, em períodos mais quentes registra-se maior retirada tanto para abastecimento público, como para irrigação das culturas, oscilando de ano para ano.

A respeito dos resultados do tempo de residência (TR), as variações estariam relacionadas ao tamanho das suas bacias de drenagem de acordo com Andrade (2010). O autor cita como resultados valores para as Lagoas Mirim e Mangueira, respectivamente, de 240 e 180 dias. Entretanto, outro fator determinante para a renovação das águas de um corpo lagunar é sua área e taxa de circulação de águas, tanto entradas quanto saídas, claramente expressas pela Equação V. Desta forma, sua interligação ou isolamento é decisivo, podendo ser exemplificado pela Lagoa dos Barros que apresentou período bem maior de renovação que as demais lagoas que são cordiformes, ou seja, possuem ligação com demais sistemas hídricos, enquanto para Barros temos apenas saídas via evaporação, infiltração e uso agrícola, maximizando a permanência das águas por mais extenso período.

Ainda, quanto ao tempo de renovação das águas, ambientes com TR mais elevados são bastante suscetíveis aos aportes antrópicos.

\section{CONCLUSÕES}

A advecção de águas subterrâneas nas lagoas costeiras do Litoral Norte do Rio Grande do Sul foi comprovada e demonstrou em todos os casos avaliados ser responsável pelo aporte de milhões de metros cúbicos por ano nestes corpos hídricos, processo importante no contexto regional.

O balanço hidrológico para cada uma das lagoas resultou em desequilíbrio entre entradas e saídas de águas, além de diferenças nas respostas entre as margens amostradas. Isso indicou possibilidade de resultados apenas parciais para os aportes, especialmente a DAS que pode ser ainda maior, bem como um comportamento caracteristicamente distinto entre a região de encosta, com processo advectivo, e a planície, com processo infiltrante. Tal desempenho, possivelmente, se justifica pela diferença entre as características geológicas de deposição, composição e consolidação de cada uma destas faixas e as forçantes envolvidas, como a influência do embasamento e a interação com o oceano.

Os períodos de renovação das águas indicam diferenças entre as lagoas amostradas, conforme suas características de interligação, resultando em tempos de residência da ordem de poucos dias até muitos meses.

É importante considerar que essa interação aquífero-lagoa deva ser acompanhada de forma a avaliar se é intensa e contínua a contribuição de água subterrânea para os corpos lagunares. Em larga escala, isso pode afetar a qualidade e os estoques dos aquíferos, comprometendo os níveis dos mesmos e, consequentemente, os níveis das lagoas pelo efeito de tamponamento hidráulico, que é a relação de equilíbrio entre o manancial subterrâneo e as lagoas, ainda pouco conhecida.

\section{AGRADECIMENTOS}

Contribuição do INCT Mar-COI 565062/2010-7 e CNPq 303672/2013-7.

\section{REFERÊNCIAS}

Andrade, C.F.F. 2010. Conexões e interações entre a água superficial e subterrânea na região costeira do extremo Sul do Brasil. Tese de Doutorado. Pós Graduação em Oceanografia Física, Química e Geológica. Instituto de Oceanografia. Universidade Federal do Rio Grande, Rio Grande. 194p.

Andrade, C.F.F.; Niencheski, L.F.H.; Attisano, K.K.; Milani, M.R.; Santos, I.R. \& Milani, I.C. 2011. Fluxos de nutrientes associados às descargas de água subterrânea para a Lagoa Mangueira (Rio Grande do Sul, Brasil). Quím. Nova XY: 1-6.

Attisano, K.K.; Niencheski, L.F.H.; Milani, I.C.B.; Machado, C.S.; Milani, M.R. \& Zarzur, S. 2008. Contribution from continental groundwater to the shelf zone in Albardão area, RS, Brazil. Braz. J. Ocean. 56: 189-200.

Barboza, E.G.; Rosa, M.L.C.C.; Hesp, P.A.; Dillenburg, S.R.; Tomazelli, L.J. \& Ayup-Zouain, R.N. 2011. 
Evolution of the Holocene Coastal Barrier of Pelotas Basin (Southern Brazil) - a new approach with GPR data. J. Coast. Res. SI 64: 646-650.

Burnett, W.C.; Bokuniewicz, H.; Huettel, M.; Moore, W.S. \& Taniguchi, M. 2003. Groundwater and pore water inputs to the coastal zone. Biogeochemistry. 66: 3-33.

Burnett, W.C.; Aggarwal, P.K.; Aureli, A.; Bokuniewicz, H.; Cable, J.E.; Charette, M.A.; Kontar, E.; Krupa, S.; Kulkarni, K.M.; Loveless, A.; Moore, W.S.; Oberdorfer, J.A.; Oliveira, J.; Ozyurt, N.; Provinec, P.; Privitera, A.M.G.; Rajar, R.; Ramessur, R.T.; Scholten, J.; Stieglitz, T.; Taniguchi, M. \& Turner, J.V. 2006. Quantifying submarine groundwater discharge in the coastal zone via multiple methods. Sci. Total Environ. 367: 498-543.

Cable, J.E.; Burnett, W.C. \& Chanton, J.P. 1997a. Magnitude and variantions of groundwater seepage along a Florida marine shoreline. Biogeochemistry. 38: 189-205.

Cable, J.E.; Burnett, W.C.; Chanton, J.P.; Corbett, D.R. \& Cable, P.H. 1997b. Field evaluation of seepage meters in the coastal marine environment. Estuar. Coast. Shelf S. 45: 367-375.

Cable, J.E.; Burnett, W.C.; Chanton, J.P. \& Weatherly, G.L. 1996. Estimating groundwater discharge into the northeastern Gulf of Mexico using radon-222. Earth Planet. Sci. Lett. 144: 591-604.

Corbett, D.R.; Chanton, J.; Burnett, W.; Dillon, K.; Rutkowski, C. \& Fourqurean, J.W. 1999. Patterns of groundwater into Florida Bay. Limnol. Oceanogr. 44: 1045-1055.

Delaney, P. 1965. Fisiografia e geologia da subsuperfície da Planície Costeira do Rio Grande do Sul. Publicação Especial. Escola de Geologia, UFRGS. Porto Alegre, n.6, 195p.

Dillenburg, S.R. \& Barboza, E.G. 2014. The strikefed sandy coast of Southern Brazil. In: Martini, I.P. \& Wanless, H.R. (eds.) Sedimentary Coastal Zones from High to Low Latitudes: similarities and differences. Geological Society, London. Special Publications 388: 333-352pp.

Gomes, S. 2005. Ajuste e avaliação de um modelo de balance hídrico decendial e estudo dos limites de sua utilização em algumas localidades no estado do Paraná. Tese de Mestrado. Pós Graduação em Agronomia. Departamento de Solos e Engenharia Agrícola. Universidade Federal do Paraná, Curitiba. 120p.

Guerra, A.J.T. \& Cunha, S.B. 1994. Geomorfologia: uma atualização de bases e conceitos. $1^{\circ}$ Edição. Bertrand Brasil, Rio de Janeiro, 458p.

Hesp, P.A.; Dillenburg, S.R.; Barboza, E.G.; Tomazelli, L.J.; Ayup-Zouain, R.N.; Esteves, L.S.; Gruber, N.L.S.; Toldo, E.E.; Tabajara, L.L.C.A.
\& Clerot, L.C.P. 2005. Beachridges, foredunes or transgressive dunefields? Definitions and an examination of the Torres to Tramandaí barrier system, Southern Brazil. An. Acad. Bras. Cienc. 77: 493-508.

INMET - Instituto Nacional de Meteorologia 2013. Banco de dados meteorológico para ensino e pesquisa. Site <http://www.inmet.gov.br/portal/ index.php?r=bdmep/b>. Acesso: 29/04/2013.

Ivanoff, M.D. 2013. Sedimentação da Lagoa da Itapeva, RS - Brasil. Dissertação de Mestrado. Pós Graduação em Geociências. Instituto de Geociências. Universidade Federal do Rio Grande do sul, Porto Alegre. 56p.

Johannes, R. 1980. The ecological significance of the submarine discharge of groundwater. Mar. Ecol.Prog. Ser. 3: 365-373.

Lee, D.R. 1977. A device for measuring seepage flux in lakes and estuaries. Limnol. Oceanogr. 22: 140-147.

Machado, J.L.F. \& Freitas, M.A. 2005. Projeto Mapa Hidrogeológico do Estado do Rio Grande do Sul: relatório final. CPRM, Porto Alegre, 65p.

Milani, E.J.; Melo, J.H.G.; Souza, P.A.; Fernandes, L.A. \& França, A.B. 2007. Bacias do Paraná. In: Milani, E.J.; Rangel, H.D.; Bueno, G.V.; Stica, J.M.; Winter, W.R.; Caixeta, J.M. \& Pessoa Neto, O.C. (eds.) Bacias Sedimentares Brasileiras Cartas Estratigráficas. Boletim de Geociências da Petrobras. Rio de Janeiro, 265-287pp.

Mulligan, A.E. \& Charette, M.A. 2006. Intercomparison of submarine groundwater discharge estimates from a sandy unconfined aquifer. J. Hydrol. 327: 411-425.

Niencheski, L.F.H.; Windom, H.L.; Moore, W.S. \& Jahnke, R.A. 2007. Submarine groundwater discharge of nutrients to the ocean along a coastal lagoon barrier, Southern Brazil. Mar. Chem. 106: 546-561.

Oberdorfer, J.A.; Valentino, M.A. \& Smith, S.V. 1999. Groundwater contribution to the nutrient budget of Tomales Bay, California. Biogeochemistry 10: 199-216.

Paiva, M.L. 2011. Sistema aquífero Guarani: fonte de nutrientes e elementos traços para a zona costeira. Monografia de Graduação. Instituto de Oceanografia. Universidade Federal do Rio Grande, Rio Grande. 93p.

Pivel, M.A.G. \& Calliari, L.J. 1998. Caracterização preliminar das praias de Torres (RS) sob um enfoque morfodinâmico. In: XI Semana Nacional de Oceanografia. Rio Grande, FURG.

Profill, E.A. 2005. $1^{\circ}$ Etapa do Plano da Bacia do Rio Tramandaí - Relatório da Fase A - Consolidação do Diagnóstico e Balanço Hídrico. SEMA/DRH- 
FEPAM, Porto Alegre, 95p.

Rebouças, A.C. 1994. Sistema Aquífero Botucatu no Brasil. In: VIII Congresso Brasileiro de Águas Subterrâneas. In: Anais do VIII Congresso Brasileiro de águas Subterrâneas, Associação Brasileira de Águas Subterrâneas. Pernambuco, 500-509pp.

Rosa Filho, E.F.; Hindi, E.C.; Rostirolla, S.P.; Ferreira, F.J.F. \& Bitencourt, A.V.L. 2003. Sistema Aquífero Guarani - considerações preliminares sobre a influência do Arco de Ponta Grossa no fluxo de águas subterrâneas. Rev. Ág. Subt. 17: 91-112.

Rosa, M.L.C.C. 2012. Geomorfologia, estratigrafia de sequências e potencial de preservação dos Sistemas Laguna-Barreira do Quaternário Costeiro do Rio Grande do Sul. Tese de Doutorado. Pós Graduação em Geociências. Instituto de Geociências. Universidade Federal do Rio Grande do sul, Porto Alegre. 251p.

Santos, I.R.; Burnett, W.C.; Chanton, J.; Mwashote, B.; Suryaputra, I.G.N.A. \& Dittmar, T. 2008a. Nutrient biogeochemistry in a Gulf of Mexico subterranean estuary and groundwater-derived fluxes to the coastal ocean. Limnol. Oceanogr. 53: 705-718.

Santos, I.R.; Niencheski, F.; Burnett, W.; Peterson, R.; Chanton, J.; Andrade, C.F.F.; Milani, I.B.; Schmidt, A. \& Knoeller, K. 2008b. Tracing anthropogenically driven groundwater discharge into a coastal lagoon from southern Brazil. J. Hydrol. 353: 275-293.

Sassaki, R.A. 2005. Distribuição de tempos de residência em sistemas alimentados com vazão variável. Dissertação de Mestrado. Pós Graduação de Engenharia. Universidade Federal do Rio de Janeiro, rio de Janeiro. 100p.

Schwarzbold, A. \& Schafer, A. 1984. Gênese e morfologia das lagoas costeiras do Rio Grande do Sul - Brasil. Amazon. 9: 87-104.

Shaw. R.D. \& Prepas, E.E. 1989. Anomalous, shortterm influx of water into seepage meters. Limnol. Oceanogr. 34: 1343-1351.

Silva, R.P.; Calliari, L.J. \& Tozzi, H.A.M. 2003. The influence of washout on the erosive susceptibility of the Rio Grande do Sul coast between Cassino and Chuí Beaches, Southern Brazil. J. Coast. Res. SI 35: 332-338.

Simmons, G. M. 1992. Importance of submarine groundwater discharge (SGWD) and seawater cycling to material flux across sediment/water interfaces in marine environments. Mar. Ecol.Progr. Ser. 84: 173-184.
Taniguchi, M.; Ishitobi, T.; Burnett, W.C. \& Wattayakorn, G. 2007. Evaluating ground water-sea water interactions via resistivity and seepage meters. Ground Water 45(6): 729-735.

Tomazelli, L.J. 1990. Contribuição ao estudo dos sistemas deposicionais holocênicos do nordeste da Província Costeira do Rio Grande do Sul - com ênfase no sistema eólico. Tese de Doutorado. Pós Graduação em Geociências. Instituto de Geociências. Universidade Federal do Rio Grande do Sul, Porto Alegre. 270p.

Tomazelli, L.J. 1993. O regime de ventos e a taxa de migração das dunas eólicas costeiras do RS, Brasil. Pesqui. 20: 18-26.

Tomazelli, L.J.; Barboza, E.G.; Rosa, M.L.C.; Dillenburg, S.R.; Caron, F.; Manzolli, R.P. \& Lima, L.G. 2011. Terraços lagunares holocênicos da margem da Lagoa do Gentil, Litoral Norte do Rio Grande do Sul: gênese e significado para a evolução geológica regional. In: Anais do XIII Congresso da Associação Brasileira de Estudos do Quaternário - ABEQUA. Armação de Búzios.

Tomazelli, L.J. \& Villwock, J. A. 2005. Mapeamento Geológico de Planícies Costeiras: o Exemplo da Costa do Rio Grande do Sul. Gravel 3: 109-115.

Valiela, I. \& D'Elia, C. 1990. Groundwater Inputs to Coastal Waters: Special Issue, Biogeochemistry 10, 328p.

Valiela, I.; Teal, J.M.; Volkmann, S.; Shafer, D. \& Carpenter, E.J. 1978. Nutrient and particulate fluxes in a salt marsh ecosystem: tidal exchanges and inputs by precipitation and groundwater. Limnol. Oceanogr. 23: 798-812.

Villwock, J.A. \& Tomazelli, L.J. 1995. Geologia Costeira do Rio Grande do Sul. Notas Técnicas do Centro de Estudos em Geologia Costeira e Oceânica, Universidade Federal do Rio Grande do Sul 8: 1-45.

Zimmermann, C.F.; Montgomery, J.R. \& Carlson, P.R. 1985. Variability of Dissolved Reactive Phosphate Flux Rates in Nearshore Estuarine Sediments: Effects of Groundwater Flow. Estuaries 8: 228-236.

Submetido: Agosto/2014

Revisado: Setembro/2015

Aceito: Outubro/2015 